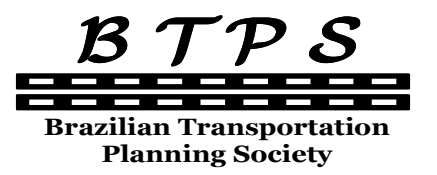

Planning Society

\author{
Journal of Transport Literature \\ Vol. 6, n. 4, pp. 133-146, Oct 2012 \\ Reviews \& Essays
}

JTL | RELIT

www.transport-literature.org ISSN 2238-1031

\title{
Eficiência portuária: análise das principais metodologias para o caso dos portos brasileiros
}

[Port efficiency: analysis of the main approaches for the Brazilian ports]

\author{
Viviane Adriano Falcão*, Anderson R. Correia \\ Instituto Tecnológico de Aeronáutica (ITA), Brazil, Instituto Tecnológico de Aeronáutica (ITA), Brazil
}

Submitted 1 May 2011; received in revised form 12 Dec 2011; accepted 26 Jan 2012

\begin{abstract}
Resumo
Sabendo que mais de $80 \%$ do comércio internacional brasileiro é realizado via marítima, o setor portuário assume uma importância indiscutível no processo de crescimento econômico nacional, inclusive como um fator de desenvolvimento sócioeconômico no seu entorno. Dentro desse contexto, a necessidade de avaliar o desempenho portuário se torna essencial para assegurar a competitividade dos produtos nacionais em mercados externos. Neste artigo, são abordadas as duas principais metodologias de avaliação de eficiência portuária, em especial a Fronteira Estocástica e a Análise Envoltória de Dados. Serão levantadas evidências do desempenho das técnicas existentes e quais se adequam melhor para o caso da avaliação da eficiência portuária. A fronteira estocástica apresentou como principal vantagem o fato de comparar os resultados dos portos analisados com parâmetros técnicos ou normalizados, porém, só consegue analisar um produto por vez. A análise envoltória de dados, por sua vez, tem como vantagem a possibilidade de analisar múltiplos produtos, porém, com um número reduzido de dados amostrais.
\end{abstract}

Palavras-Chave: portos; eficiência portuária; análise envoltória de dados; fronteira estocástica.

\begin{abstract}
More than $80 \%$ of the Brazilian international trade is by sea, the port assumes an unquestionable importance in the process of national economic growth. Also considered as a factor for socioeconomic development to region nearby. In this context, the necessity to evaluate the port efficiency is essential to assure the competitiveness of the national products on external markets. This paper will analyze the two main approaches of evaluation of port efficiency, in special the Stochastic Frontiers and the Data Envelopment Analysis. It will show the main evidences of the performance of the existing techniques and which is the better for the case of the port efficiency. The Stochastic Frontiers presented as main advantage the fact to compare the results with technical parameters or normalized, however it only analyze one product for time. The Data Envelopment Analysis has as advantage, the possibility to analyze multiples products, however with a reduced number of samples.
\end{abstract}

Key words: ports; port efficiency; data envelopment analysis; stochastic frontier.

* Corresponding Author. Email: viviane.falcao@yahoo.com.br.

\section{Recommended Citation}

Falcão, V. A. and Correia, A. R. (2012) Eficiência portuária: análise das principais metodologias para o caso dos portos brasileiros. Journal of Transport Literature, vol. 6, n. 4, pp. 133-146.

- JTL/RELIT is a fully electronic, peer-reviewed, open access, international journal focused on emerging transport markets and published by BPTS - Brazilian Transport Planning Society. Website www.transport-literature.org. ISSN 2238-1031.

This paper is downloadable at www.transport-literature.org/open-access. 


\section{Introdução}

Desde a época das grandes navegações, que teve início no século $\mathrm{XV}$, o transporte marítimo tem uma considerável importância na economia mundial. Atualmente, com o advento da globalização o transporte marítimo e, consequentemente, os portos, se tornaram peças fundamentais para alavancar o desenvolvimento do comércio internacional de um país. Até porque está mais do que confirmado que a maior parte do comércio internacional é realizado via marítima.

Dentro desse contexto, nasce a preocupação mundial em estudar maneiras de otimizar os custos da infraestrutura portuária e, por conseguinte, ganhar competitividade. Os portos são considerados como uma das principais forças que movem a economia. Ao diminuir os custos relacionados a ele, a economia global ganha.

No Brasil, existe uma crescente preocupação com a eficiência portuária, sobretudo pelo fato que o "Custo Brasil" (conjunto de fatores desfavoráveis que encarecem o investimento no Brasil) afeta diretamente na competitividade e na eficiência da indústria nacional além de ser bastante influenciado por problemas relacionados aos portos nacionais. De acordo com a Associação Nacional dos Usuários de Transportes de Carga (ANUT) apud Dos Santos e Haddad (2007) os atrasos no embarque e desembarque nos portos, causados, entre outros, pelo número insuficiente de berços e contêineres e pelo tamanho inadequado dos berços, custou ao Brasil US\$ 1,2 bilhões em 2004.

Segundo informações do MDIC (Aliceweb), considerando dados analisados entre 2005 e 2010 as exportações via marítima representam em média $82 \%$ de toda a exportação brasileira. Em 2009 e 2010 as exportações brasileiras aumentaram de 32\% enquanto que as exportações via marítima aumentaram de $34 \%$. No tocante as importações, também se percebe uma importância considerável das importações via marítima correspondendo a aproximadamente $70 \%$ considerando os dados entre 2005 e 2010. Entre 2009 e 2010 obteve-se um crescimento de $42 \%$ nas importações totais e cerca de $48 \%$ nas importâncias via marítima.

Segundo o relatório Global Competitiviness Report 2010 - 2011, em termos de qualidade da infraestrutura portuária, o Brasil ocupa centésima vigésima terceira posição, atrás de todos os 
países do BRIC (Brasil, Rússia, Índia e China) e também, atrás da Argentina, México, Nigéria, Polônia, Paraguai e Colômbia.

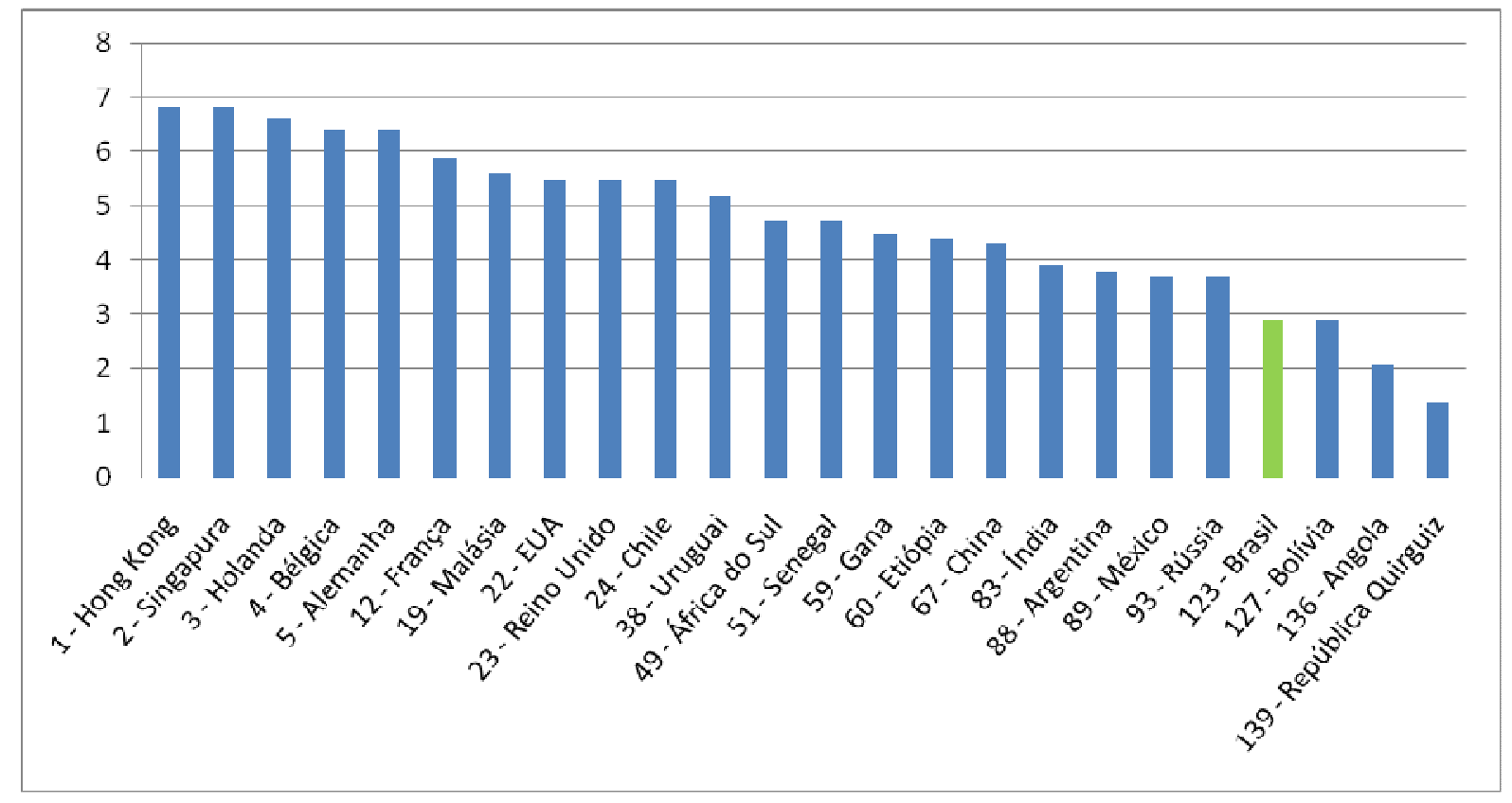

Figura 1 - Índice de Qualidade de Infra-estrutura Portuária.

Fonte: Schwab (2010).

As informações e considerações acima citadas confirmam a importância dos Portos para economia brasileira. Dentro desse contexto, o objetivo principal desse artigo é fazer um levantamento e uma breve análise das principas técnicas e metodologias existentes para avaliação eficiências portuárias. Neste artigo, são abordadas as duas principais metodologias de avaliação de eficiência portuária, em especial a Fronteira Estocástica e a Análise Envoltória de Dados. Serão levantadas evidências do desempenho das técnicas existentes e quais se adequam melhor para o caso da avaliação da eficiência portuária.

A metodologia da fronteira estocástica apresentou como principal vantagem o fato de comparar os resultados dos portos analisados com parâmetros técnicos ou normalizados, porém só consegue analisar um produto por vez. A análise envoltória de dados, por sua vez, tem como vantagem a possibilidade de analisar múltiplos produtos, porém com um número reduzido de dados na amostra a fim de não causar distorções nos resultados.

O presente trabalho está dividido da seguinte forma, a seção 1: Introdução, nela foram apresentados as principais diretrizes do trabalho: problemática, importância, objetivo, e a 
estrutura do trabalho sob forma de artigo científico. A seção 2: Portos onde tem uma revisão bibliográfica de Portos no seu contexto geral. A seção 3: Principais métodos de avaliação da Eficiência Portuária que é uma revisão bibliográfica das técnicas mais utilizadas na avaliação da Eficiência Portuária, que sejam as mais importantes a Análise Envoltória de Dados e as Fronteiras Estocásticas. Por fim, tem-se a conclusão.

\section{Portos}

Conforme Collyer apud Sousa Jr., (2010) pode-se definir porto como entreposto dinâmico de mercadorias, em que se realizam atividades (aduaneiras, alfandegárias, comerciais, sanitárias, tributárias, imigratórias etc.). É a porta de entrada e saída de mercadorias e passageiros, servindo de abrigo e ancoradouro das embarcações, além de estar munido de instalações necessárias para o embarque e desembarque de cargas e passageiros e, sobretudo, o mais importante elo da cadeia logística que supre a humanidade.

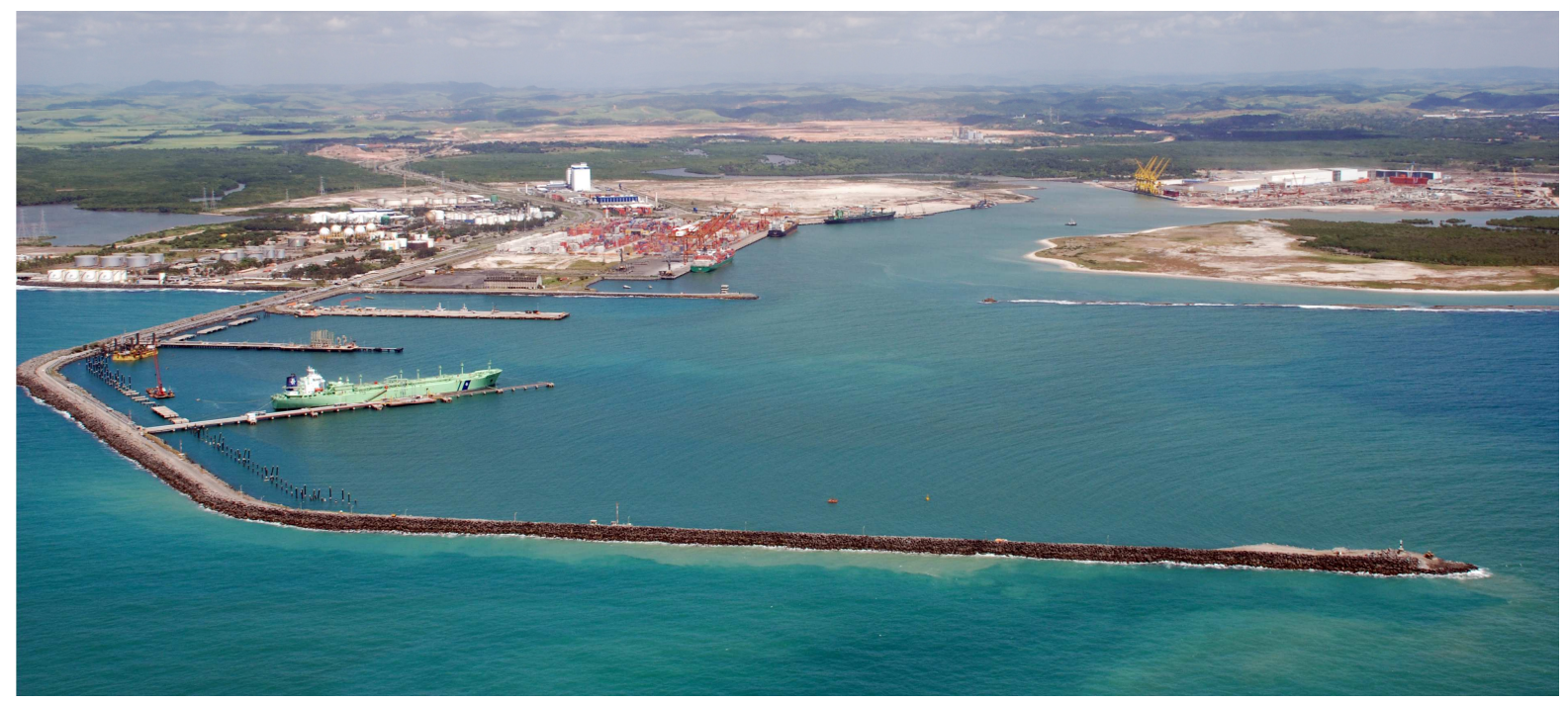

Figura 2 - Complexo Industrial Portuário de Suape - PE. Fonte: Panoramio.com

O porto é considerado um elo na cadeia de transporte, sendo assim, sua principal finalidade é promover o desenvolvimento econômico de uma região através do escoamento de pessoas, de bens e de mercadorias e, assim, movimentar a economia. Os transportes existem para facilitar o deslocamento de pessoas e bens no espaço e no tempo. Pode-se considerar os seus principais objetivos a promoção do desenvolvimento sócio-econômico, romper barreiras, permitindo assim o escoamento e a comercialização de bens e serviços. 
As cargas nos portos podem ser classificadas em três tipos: granel sólido, granel líquido e carga geral. Alumina, carvão, minério de ferro, soja, trigo e sal são exemplos de remessas classificadas como granel sólido. Os derivados do petróleo, gases liquifeitos e produtos químicos em geral são considerados granéis líquidos. Produtos diversos, como frutas, produtos agrícolas e carnes são considerados como carga geral. Estes ainda tem uma subclassificação, pode ser considerada solta ou conteinerizada.

Segundo Branch apud Esmer (2008), os portos tem como objetivos principais:

a) Embarque e desembarque de cargas e de passageiros.

b) Proporcionar serviços de ancoragem e reparo para navios.

c) Fornecer abrigo para navios em dias de tempestade.

d) Fundamental para o desenvolvimento industrial.

e) Fazer parte da cadeia de transporte.

Pode acrescentar também, como objetivos de segundo grau, a armazenagem de mercadoria, despachos aduaneiros, reconhecimento de avarias, entre outros.

A UNCTAD apud Sousa Jr., Ferreira Jr. \& Prata (2008) classifica os portos por geração, sendo o mais antigo considerado como porto de $1^{\mathrm{a}}$ geração e o mais moderno como porto de $4^{\mathrm{a}}$ geração. 
Tabela 1 - Classificação da UNCTAD para portos. Adaptado de Sousa Jr., Ferreira Jr. \& Prata (2008)

\begin{tabular}{|c|c|}
\hline GERAÇÃO & DEFINIÇÃO GERAL E ALGUMAS CARACTERÍSTICAS \\
\hline \multirow{7}{*}{ 1a. } & Interface entre dois modos de transporte \\
\hline & Não existe desenvolvimento estratégico específico \\
\hline & Atividades tradicionais de manuseio e armazenagem não organizadas \\
\hline & Atividades localizadas nas docas \\
\hline & Justaposição das relações entre os portos \\
\hline & Supremacia do suprimento \\
\hline & Pouca atenção dada às necessidades dos usuários \\
\hline \multirow{6}{*}{$2 \mathbf{a}}$. & Centro de transporte, atividades industriais e comércio \\
\hline & Surgimento e expansão do desenvolvimento estratégico \\
\hline & Atividades de transformação (indústrias pesadas), serviços de navios \\
\hline & Ampliação da zona portuária \\
\hline & Início de uma comunidade portuária \\
\hline & Relacionamento ocasional entre o porto e sua cidade adjacente \\
\hline \multirow{7}{*}{ 3a. } & $\begin{array}{l}\text { Centro de transporte integrado com plataforma logística para o comércio } \\
\text { internacional }\end{array}$ \\
\hline & Desenvolvimento estratégico orientado ao Mercado \\
\hline & Distribuição de mercadorias, atividades logísticas, centro de distribuição \\
\hline & $\begin{array}{l}\text { Sistema de Informação (EDI - Electronic Data Interchange - conjunto de formatos } \\
\text { de documentos eletrônicos para transações entre organizações) utilizado pelo porto }\end{array}$ \\
\hline & Racionalização do espaço do porto \\
\hline & Comunidade portuária unificada e ativa, coordenação das atividades \\
\hline & Forte relacionamento entre porto e cidade \\
\hline \multirow{6}{*}{ 4a. } & Portos em rede \\
\hline & $\begin{array}{l}\text { Estratégia para tornar-se um membro da rede mundial; diversificação das } \\
\text { atividades }\end{array}$ \\
\hline & Parceria com operadores na organização dos serviços logísticos \\
\hline & Uso das redes EDI integradas entre os portos \\
\hline & $\begin{array}{l}\text { Participação em pesquisa de locais para portos, visando possível desenvolvimento } \\
\text { e integração }\end{array}$ \\
\hline & Cooperação entre as comunidades portuárias \\
\hline
\end{tabular}

Segundo Gonzalez e Trujillo (2008) os portos tem um importante papel na cadeia logística, sendo assim o nível de eficiência portuária influencia, enormemente, a competitividade de um país, por conseguinte uma alta eficiência portuária conduz a baixas tarifas de exportações que, por sua vez, favorecem a competitividade dos produtos nacionais em mercados internacionais. A fim de manter uma posição de competidor no mercado internacional, os países precisam trabalhar nos fatores que influenciam a eficiência de seus portos. 
Segundo UNCTAD apud Sousa Jr., (2010) em termos de planejamento portuário é essencial destacar três atividades principais:

i. Preparação do plano nacional dos portos: definição da função de cada porto no âmbito nacional priorizando a otimização na utilização dos recursos nacionais.

ii. Preparação do plano geral de cada porto: estabelecimento das modalidades do desenvolvimento do porto em longo prazo, sem preocupação com as datas.

iii. Preparação de projetos portuários: por em prática cada parte do plano geral, com definição das datas.

Ainda conforme UNCTAD apud Sousa Jr., (2010), as funções do porto podem ser divididas em externas e internas.

Classificação das funções externas:

i. Funções destinadas aos navios: os serviços prestados aos navios logo que chegam ao porto, esses serviços são realizados pelos práticos e rebocadores.

ii. Funções realizadas na "interface" mar-terra: O manuseio da carga ocorre nesse grupo. Nessa etapa, a fim de obter eficiência, é necessário o uso dos sistemas operacionais integrados. Esses sistemas devem ser empregados em todas as operações executadas, desde o porão dos navios até a saída das embarcações do porto.

iii. Funções em terra: Os serviços deste grupo são realizados em terra. Consistem na prestação dos mais variados serviços, correspondentes às necessidades de eficiência e qualidade impostas pela concorrência. Como exemplo de atividades, tem-se o manuseio de cargas do ponto de atracação até os pátios e armazéns.

Em termos de funções internas tem-se: as funções econômicas, físicas, financeiras, sociais, comerciais e de desenvolvimento.

Pelo que foi escrito acima e conforme Esmer (2008) há muitas razões pelas quais um porto precisa medir o seu desempenho, entre elas: 
- Primeiramente, precisa saber se o mesmo está operando com eficácia. Quanto carga é manipulada diariamente? Quantos clientes por semana?

- Em seguida, deve saber quanto eficientemente se está operando. Que recursos (em termos de pessoal, de máquinas, de superfície etc.) são necessários para realizar suas atividades? Quanto de carga movimentada é considerada por empregado? Quanto custa para movimentar cada tonelada da carga?

- É necessário também, saber confrontar o desempenho atual com o desempenho do porto no passado. Está movimentando mais carga por empregado ou por máquina do que no ano passado? Há alguma melhoria na eficiência?

- Assim como qualquer negócio precisa de objetivos, os portos também precisam e deve-se comparar o seu desempenho com aqueles objetivos traçados anteriormente. $\mathrm{O}$ porto conseguiu atingir os objetivos de produção colocados no início do ano? Alcançou seus objetivos de trafegabilidade?

- É de suma importância que um porto ou um terminal compare seu desempenho com o dos seus concorrentes.

- Considerando o seu desempenho atual, é essencial ajustar seus objetivos para o futuro.

- Finalmente, é importante promover seu negócio e atrair novos clientes. Assim como é do seu interesse monitorar constantemente a satisfação dos seus clientes.

\section{Principais métodos de avaliação da eficiência portuária}

Neste subcapítulo pretende-se abordar os principais trabalhos, que foram estudados, sobre métodos de cálculo da eficiência portuária. O objetivo é fazer um breve resumo sobre os principais trabalhos nesse domínio.

Conforme Gomes e Ponchio. apud Acosta et al. (2011), a eficiência técnica de uma unidade produtiva é pode ser medida pela razão entre a produção observada e a produção máxima, ou pela razão entre a quantidade mínima necessária de recursos e a quantidade efetivamente empregada, dada a quantidade de produtos gerada. 
Uma medida de eficiência pode ser descrita como:

$$
\text { Eficiência }=\frac{\text { soma ponderada dos produtos }}{\text { soma ponderada dos insumos }}
$$

Segundo Lima Jr. apud Sousa Jr., Ferreira Jr. \& Prata (2008), pode considerar quatro passos básicos para a estruturação da medição de desempenho:

i. Definição de quais atributos, tais como, tempo, custo, nível de serviço, qualidade, são considerados críticos para que o sistema atinja seus objetivos;

ii. Mapeamento dos processos interfuncionais usados para obter resultados e identificação das relações de causa e efeito existentes;

iii. Identificação dos elementos críticos e das capacidades necessárias para a execução dos processos satisfatoriamente; e

iv. Concepção de medidas que avaliem esses elementos e capacidades, assim como os respectivos padrões e metas.

Em se tratando de portos, a complexidade e a heterogeneidade das suas operações dificultam o uso de ferramentas analíticas como método de avaliação. Sendo assim, é preferível centrar a análise da eficiência portuária em uma atividade em particular. Dentro desse contexto, UNCTAD apud Esmer (2008) classifica os indicadores de desempenho portuários em duas classes principais, financeira e operacional.

As principais metodologias utilizadas na avaliação da eficiência portuária podem ser separadas em dois grupos principais. No primeiro grupo estão os trabalhos que avaliam a eficiência relativa dos portos, do tipo Análise Envoltória de Dados (DEA - Data Envelopment Analysis). No segundo grupo estão os trabalhos que consideram a eficiência técnica ou econômica dos portos, seria o grupo do tipo fronteiras estocásticas (SFA - Stochastic Frontier Analysis).

Vale salientar que mesmo quando o estudo é centrado sobre uma variável específica, ainda existe diversidade, afinal o porto rende serviços tanto aos navios como aos passageiros e à carga. A carga, propriamente dita, não deve ser considerada como homogênea, pois cada tipo 
de produto tem necessidade de equipamentos específicos para o carregamento/descarregamento, como por exemplo, os contêneires usam guindastes especializados, os líquidos utilizam sistemas de tubulação, entre outros.

\subsection{Fronteiras Estocásticas}

A avaliação baseada em Fronteiras Estocásticas SFA é uma metodologia paramétrica e estocástica. O SFA, por ser paramétrico, é definido como um modelo descrito a partir de uma equação linear, não utiliza dimensões restritas, não tem restrição quanto ao tamanho da amostra e os dados tanto podem ter baixa quanto alta correlação.

A metodologia SFA faz uma comparação do desempenho dos portos com padrões técnicos ou comportamentais. A principal desvantagem desse método é a necessidade de um grande número de dados de difícil acesso, além dos problemas com as definições dos insumos e produtos das atividades dos portos.

Segue abaixo, um exemplo de uma função SFA para a avaliação de eficiência no caso dos portos, pode perceber claramente a complexidade da mesma, Estache, A., et al. (2002).

$$
\begin{gathered}
\ln Q_{i t}=\beta_{0}+\beta_{1} \ln K_{i t}+\beta_{2} \ln L_{i t}+\beta_{3} t+\beta_{4} \ln \left(K_{i t}\right)^{2}+\beta_{5} \ln \left(K_{i t}\right) \ln \left(L_{i t}\right)+\beta_{6} \ln \left(K_{i t}\right) t+\beta_{7} \ln \left(L_{i t}\right)^{2}+ \\
\beta_{8} \ln \left(L_{i t}\right) t+\beta_{9} t^{2}+v_{i t}-u_{i t},
\end{gathered}
$$

Onde:

as variáveis são todos desvios geométricos e definidos como : $\mathrm{i}=1, \ldots, \mathrm{N}$ e $\mathrm{t}=1, \ldots, \mathrm{M}$

$\mathrm{Q}_{\mathrm{it}}$ é o volume de movimentação de mercadorias do porto "i” durante um período " $\mathrm{t}$ "

$\mathrm{K}_{\mathrm{it}}$ é o capital usado pelo porto "i" durante um período " $\mathrm{t}$ ",

$\mathrm{L}_{\mathrm{it}}$ é a quantidade de empregados no porto "i” durante um período " $\mathrm{t}$ ",

té o tempo

$\mathrm{V}_{\text {it }}$ é o erro aleatório

$\mathrm{u}_{\mathrm{it}}$ é a variável aleatória não negativa associada com ineficiência técnica 


\subsection{Análise Envoltória de Dados}

As principais características da Análise Envoltória de Dados - DEA, por ser um modelo não paramétrico e determinístico, é ser uma técnica baseada na programação linear. O DEA, não apresenta coeficientes, e quanto menor a amostra, melhores serão as interpretações, porém há necessidade de escolher uma amostra correlacionada para obter informações mais precisas. Uma importante característica desse método é a sua flexibilidade, quanto a sistemas com diversos insumos e produtos.

É importante salientar que no DEA, é analisado, somente, uma unidade por vez. A metodologia DEA, mede o desempenho relativo dos portos, isso significa que obtem-se resultados e os comparam entre eles mesmos para posteriormente ordená-los. A principal deficiência é a necessidade de um grande número de dados de difícil acesso.

A seguir é apresentada a equação do modelo DEA, conforme Sousa Junior, J. N. C. (2010).

$$
\begin{gathered}
M A X z_{0}=\frac{\sum_{y=1}^{O} v_{y} O_{y 0}}{\sum_{x=1}^{O} u_{x} I_{x 0}} \\
\frac{\sum_{y=1}^{O} v_{y} O_{y k}}{\sum_{x=1}^{O} u_{x} I_{x k}} \leq 1, \forall k \\
u_{x}, v_{y} \geq 0, \forall x, y
\end{gathered}
$$

Onde,

$z_{0}$ : Eficiência da DMU (Unidade de Tomada de Decisão) 0;

I: número total de inputs;

$O:$ número total de outputs;

$n$ : número total de DMU (Unidade de Tomada de Decisão);

Ixk: quantidade de input $x$ para DMU $k ; k=1,2, \ldots, n$;

Oyk: quantidade de output y para DMU $k ; k=1,2, \ldots, n$;

$u x$ : peso outorgado ao input $x$;

$v y$ : peso outorgado ao output $y$. 


\subsection{Diferenças entre os métodos de avaliação de Eficiência Portuária}

Para demonstrar as principais diferenças entre as metodologias apresentadas, Fronteiras Estocásticas e Análise Envoltória de Dados, foi elaborado uma tabela adaptada de Gonzalez \&Trujillo (2008) conforme abaixo.

Tabela 2 - Principais características do DEA e do SFA. Adaptado de Gonzalez \&Trujillo.

\begin{tabular}{|l|l|}
\hline Análise Envoltória de Dados-DEA & Fronteiras Estocásticas - SFA \\
\hline Metodologia não paramétrica & Metodologia paramétrica \\
\hline Metodologia determinística & Metodologia estocástica \\
\hline $\begin{array}{l}\text { Não permite que a hipótese } \\
\text { estatística seja comparada }\end{array}$ & $\begin{array}{l}\text { Permite que a hipótese estatística seja } \\
\text { comparada }\end{array}$ \\
\hline $\begin{array}{l}\text { Não realiza suposições na } \\
\text { distribuição do termo da ineficiência }\end{array}$ & $\begin{array}{l}\text { Realiza suposições na distribuição do } \\
\text { termo da ineficiência }\end{array}$ \\
\hline Não inclui o erro como termo & Inclui um termo composto do erro \\
\hline $\begin{array}{l}\text { Não exige a especificação de uma } \\
\text { função }\end{array}$ & Exige a especificação de uma função \\
\hline Pequeno número de variáveis & $\begin{array}{l}\text { Pode confundir ineficiência caso o } \\
\text { modelo tenha sido mal definido }\end{array}$ \\
\hline Método: Programação linear & Método: Econométrico \\
\hline
\end{tabular}

\section{Conclusão}

A importância dos Portos para economia brasileira é indiscutível. Cerca de $85 \%$ das suas exportações/importações acontecem via marítima. O Brasil, apesar de ser a $8^{a}$ economia do mundo, tem uma medíocre classificação em termos de qualidade de infraestrutura portuária, ficando atrás de economias de menor influência no mercado mundial. Sendo assim, se torna evidente a necessidade de um maior investimento nessa área.

Dentro desse contexto, e conforme Gonzalez e Trujillo (2008), como os portos tem um importante papel na cadeia logística, o nível de eficiência portuária influencia, enormemente, a competitividade de um país. Por conseguinte, uma alta eficiência portuária conduz a baixas tarifas de exportações que, por sua vez, favorecem a competitividade dos produtos nacionais em mercados internacionais. A fim de manter uma posição de competidor na mercado internacional, os países (governos) precisam melhorar os fatores que influenciam a eficiência de seus portos. 
No caso dos portos, pode-se concluir que pelo fato das suas características multi-modal, com multi-atividades e, consequentemente complexo, não existe um método, que sozinho, possa fazer uma análise global do desempenho do mesmo. A união dessas duas técnicas pode ser um avanço para determinação de eficiência. As principais técnicas podem ser classificadas em paramétricas, principalmente a Fronteira Estocástica e não paramétricas representada pela Análise Envoltória de Dados, ambas com suas vantagens e desvantagens em se tratando de eficiência portuária.

A fronteira estocástica apresentou como principal vantagem o fato de comparar os resultados dos portos considerados com parâmetros técnicos ou normalizados, porém só consegue analisar um produto por vez.

A análise envoltória de dados por sua vez, tem como vantagem, a possibilidade de considerar múltiplos produtos, porém com um número reduzido de dados na amostra a fim de não causar distorções nos resultados.

Em termos de trabalhos nacionais, assim como internacionais, percebeu-se a tendência em utilizar o método de análise envoltória de dados DEA, para avaliar a eficiência portuária, provavelmente pela facilidade em obter dados e por ter como característica a possibilidade de analisar produtos e insumos múltiplos.

Concluí-se que uma união entre as metodologias DEA e SFA constitua o primeiro passo para o nascimento de um método apropriado à realidade portuária. Devido a importância do tema para o crescimento econômico e social do país se faz necessário um estudo mais aprofundado das duas técnicas e a sua consequente aplicação em trabalhos futuros.

No caso da análise da malha portuária brasileira uma das principais críticas aos trabalhos atuais é o fato de não existir um comparativo entre os nossos portos nacionais com outros portos "internacionais" que são considerados de ponta e eficientes. Isso é de suma importância, principalmente no caso da técnica DEA, visto que a mesma faz um comparativo entre portos. 


\section{Referências}

Almeida M., Rebelatto D. (2006) Sistematização das técnicas para avaliar a eficiência: variáveis que influenciam a tomada de decisão estratégica. 12p. II SEGeT - Simpósio de Excelência em Gestão e Tecnologia.

Acosta, C. M. M., Silva, A. M. V. A. e Lima, M. L. P. (2011) Aplicação de análise envoltória de dados (DEA) para medir eficiência em portos brasileiros. Journal of Transport Literature, vol. 5, n. 4, pp. 88-102.

Barros C.P., Decomposing Growth in Portuguese Seaports:A Frontier Cost Approach. Maritime Economics \& Logistics (2005) 7, 297-315.

Bertoloto, R. F. e Mello, J. C. C. B. S. (2011) Eficiência de portos e terminais privativos brasileiros com características distintas. Journal of Transport Literature, vol. 5, n. 2, pp. 4-21.

Collyer, W. O. (2013) A importância do direito marítimo e da regulação dos transportes e portos para o desenvolvimento da logística. Journal of Transport Literature, Vol. 7, n.1.

Dos Santos R. A. C.; Haddad E. A. (2007); Eficiência relativa dos portos brasileiros: uma análise regionalizada. Área Anpec: Área 9 - Economia Regional e Urbana. Classificação JEL: R1, F14, R15.

Estache, A., Gonzalez, M., Trujillo, L., (2002) ; Efficiency gains from port reform and the potential for yardstick competition: Lessons from Mexico. World Development 30 (4), 545-560.

Gonzalez M. M. \& Trujillo L. Efficiency Measurement in the Port Industry: A Survey of the Empirical Evidence. $34 p$

Gonzalez M. M. \& Trujillo L. Reforms and infrastructure efficiency in Spain's container ports. Transportation Research Part A 42 (2008) 243-257.

Santos, J. T. A. N., Cardoso, P. e Moita, M. H. V. (2011) Atributos de Paralisação como Indicador de Produtividade da Operação Portuária de Cabotagem em Manaus. Journal of Transport Literature, vol. 5, n. 4, pp. 70-87.

Schwab, K. (2010), The Global Competitiveness Report 2010-2011, World Economic Forum.

Soner Esmer. Performance Measurements of Container Terminal Operations.18p

Sousa Junior, J. N. C. (2010). Avaliação da eficiência dos portos utilizando análise envoltória de dados: estudo de caso dos portos da região nordeste do Brasil. Fortaleza, 2010. Dissertação de Mestrado. Programa de Mestrado em Engenharia de Transportes, Universidade Federal do Ceará, Fortaleza, CE, 89 fls.

Sousa Jr., Ferreira Jr. \& Prata; Análise da eficiência dos portos da região Nordeste do Brasil baseada em Análise Envoltória de Dados. SISTEMAS \& GESTÃO, v.3, n. 2, p.74-91, maio a agosto de 2008. 